\title{
Outcome and Management of Sepsis at RozhHalat Emergency Hospital in Erbil -Kurdistan region of Iraq
}

\author{
Samir Qader Ahmad \\ Department of Emergency Medicine \\ RozhHalat Emergency Hospital \\ General Directorate of Health \\ Erbil, Kurdistan Region, Iraq \\ Samir.kurd@ymail.com
}

\author{
Shakawan Muhamad Ismaeel \\ Department of Emergency Medicine \\ Kurdistan Board for Medical Specialties \\ Ministry of Higher Education and Scientific Research \\ Erbil, Kurdistan Region, Iraq \\ shakawan56@aol.com \\ Halgurd Fathulla Ahmed \\ Department of Internal Medicine \\ Kurdistan Board for Medical Specialties \\ Ministry of Higher Education and Scientific Research \\ Erbil, Kurdistan Region, Iraq \\ fhalgurd@gmail.com
}

\author{
Dara Ahmed Mohammed \\ Department of Anatomy \\ College of Medicine \\ University of Sulaimani \\ Sulaimani, Kurdistan Region, Iraq \\ Dara.marif@univsul.edu.iq
}

\begin{abstract}
Sepsis is a very common condition in emergency hospitals and presentation is variable, it is under diagnosed with very high mortality rate. All patients with infection are at risk of developing sepsis. Sepsis is a complex condition characterized by activation of inflammatory process and coagulation system in response to microbial insult. An observational prospective study was carried out at RozhHalat emergency hospital in Erbil-Kurdistan region of Iraq between February 2017 to January 2018.The study was approved by the scientific and ethical committee of Kurdistan board of medical specialties. 50 patients aged between $(10$ - 80) years old presented to this hospital with sign and symptoms of infection were recruited to this study. 50 patients with signs and symptoms of sepsis were recorded in this study. The mean age was 47 with male to female ration of 2:3 (42\% male and 58\% female). The overall mortality rate was high at $68 \%$ with higher rate among female and older age group. The blood culture was positive in $74 \%$ of cases of which $52 \%$ were gram positive and $22 \%$ were gram-negative microorganisms. A Positive blood culture associated with higher mortality rate of $81 \%$ compared with $21 \%$ if the blood culture was negative. In majority of cases the site of infection was from multiple source (34\%) followed by respiratory infection (26\%).Skin and soft tissue infection was associated with the lowest mortality of 2.9\%. High number of cases (82\%) developed complications and $52 \%$ of cases developed more than 2 organ failures. All patients received empirical antibiotic therapy however $46 \%$ of cases received the wrong antimicrobials. The survival rate was higher
\end{abstract}

(44\%) among patients given the right antibiotics. Similarly patients had better chance of survival if appropriate fluid resuscitation therapy was give. The overall mortality among vasopressor treated patients were $72 \%$ compared with $78 \%$ chance of mortality if they were not treated with any vasopressor therapy, among those who received inotropic support Noradrenaline associated with higher survival rate(72\%). A qSOFA score of more than 2 associated with $86.4 \%$ of mortality compared with $75.5 \%$ mortality with similar SIRS criteria. Factors associated with high mortality were: female gender, older age group, positive blood culture, wrong antibiotics therapy, less fluid resuscitation, multisource of infection, multi-organ failure, high lactic acid level and high qSOFA score. This study shows that sepsis is associated with high overall mortality rate of $68 \%$ in the RozhHalat emergency hospital and higher rate of death among female and older age group. Negative blood culture, appropriate use of antibiotics and fluid therapy associated with better chance of survival. Vasopressor therapy did not result in better survival outcome except for Noradrenaline. The qSOFA score is as good as SIRS criteria in predicting mortality. High lactate, multi-organ failure and multisource of infection associated with the worst outcome.

Keywords: Sepsis, Blood culture, Lactic acid, Vasopressor.

\section{INTRODUCTION}

Sepsis is defined as life-threatening organ dysfunction caused by a dysregulated host response to an infection. 
Probably the most often related article on the epidemiology of sepsis is the 2001 publication by Angus and colleagues, which used administrative data to estimate that there were 751,000 cases $(3.0$ per 1,000 population) in the United States each year, resulting in more than 200,000 deaths [1]. More recent research suggests that sepsis causes or contributes to between one-third and one-half of all deaths occurring in hospitals in the United States, with the majority of patients presenting to hospital with sepsis rather than acquiring sepsis in hospital [2].

All available data confirm that sepsis is a major public health problem. In the most recent report, published in 2015, sepsis is considered a cause of death from an infection and death being attributed to the infection that initiated sepsis if not recognized [3]. There is a gradual increase in the frequency of hospital-based sepsis. Extreme age groups, patients with chronic disease, common use of immunosuppressive drugs, and invasive procedures for diagnosis or treatment purposes like central lines or mechanical ventilation, immunocompromised diseases like carcinoma and AIDS increase the frequency of sepsis. Hospitalization for a long time especially ICU admission has more risk to complicated sepsis and septic shock [4]. The definition of sepsis was updated in 2016 following publication of the Third International Consensus Definitions for Sepsis and Septic Shock (Sepsis-3) [5].

The 2016 consensus definitions also recommend that the Sequential (Sepsis-related) Organ Failure Assessment (SOFA) criteria and "quick" (q)SOFA criteria to be used to identify sepsis, in place of the currently used systemic inflammatory response syndrome (SIRS) criteria, which was the basis for the previous definition of sepsis [5] [6]. SOFA required multiple laboratory investigations and it is an intensive care unit (ICU) dependent mortality score based on respiratory, cardiovascular, hepatic, renal, and neurological parameters.

The qSOFA is a rapid, shortened version of SOFA for quick sepsis assessment, which designed for use outside the ICU, and parameters of this score are:

1. Respiratory rate of 22 breaths / $\mathrm{min}$ and more,

2. Systolic blood pressure of $100 \mathrm{mmHg}$ and less,

3. Altered mental state with a Glasgow Coma scale (GCS) of 14 and less.

The SOFA score is useful in predicting the clinical outcome and effective method to describe organs dysfunction in critically ill patients [7].

The Third international consensus (Sepsis-3) definitions state that the term "severe sepsis" doesn't necessarily need to be used and no longer recommended. Septic shock has also been redefined as a subset of sepsis in which profound circulatory, cellular, and metabolic abnormalities are associated with a greater risk of mortality than with sepsis alone [5]. Therefore, in sepsis 3 guidelines, patients with sepsis are categorized into 3 groups of: infections, sepsis and septic shock.

In 2016, the UK National Institute for Health and Care Excellence (NICE) published guidance on the recognition, diagnosis, and early management of sepsis and categorize patients into 3 groups according to severity of illness or death from sepsis: high risk; moderate to high risk; or low risk [4].
Depending on guideline of management and outcome of sepsis we can deal with basic therapies that should be completed in the first 24 hour of recognition of sepsis. The following sepsis therapy protocol is known as sepsis 6 resuscitation bundles:

1. High flow oxygen to maintain oxygen saturation more than $94 \%$

2. Taken blood culture before initiation of antibiotic therapy

3. Intravenous empirical antimicrobial therapy

4. Intravenous fluid resuscitation

5. Serum lactate level measurement

6. Monitoring urine output hourly [8].

Blood culture should be obtained before starting empirical antimicrobial therapy to isolate microorganism that caused infection and to detect proper antimicrobial therapy [9].

Early administration (within one hour) of appropriate antimicrobials is central to management of sepsis or septic shock. Each hour delay in administration of appropriate antimicrobials is associated with significant increase in mortality [10].

Earliest possible administration of appropriate IV antimicrobials following diagnosis of sepsis or septic shock causes best result in outcomes [11].

Broad spectrum antimicrobial therapy must be given in order to cover all likely pathogens, the choice of empirical antimicrobial therapy depends on patient's history, clinical status and local epidemiological

factors.

Key patient factors include site of infection, concomi tant underlying diseases, chronic

organfailures, medications, indwelling devices, the pre sence of immunosuppression or other form of immunocompromised, recent known infection and

Receiving antimicrobials within the previous three months [12].

Fluid resuscitation is another crucial line for management of sepsis and septic shock, sepsis especially in case with tissue hypo perfusion may be manifested by acute organ dysfunction and/or decreased blood pressure and increased serum lactate [13].

Initial fluid resuscitation begins with $30 \mathrm{~mL} / \mathrm{kg}$ of crystalloid within the first 3 hours. This fixed volume of fluid enables clinicians to initiate resuscitation while obtaining more specific information about the patient and while awaiting more precise measurements of hemodynamic status [14].

Serum lactate is a sensitive marker to defined tissue hypoxia and it is a more objective indicator for tissue perfusion as compare with physical examination or urine output. An increased in serum lactate indicates more severe sepsis and associated with increase in mortality $[15,16]$.

International guidelines recommend norepinephrine and dopamine regarded as first line vasopressor agents in septic shock. phenylephrine, epinephrine, vasopressin and terlipressin are considered second line agents. Norepinephrine shows improved survival and has less adverse effect and hemodynamically more beneficial than dopamine because of these Norepinephrine remain 
as a first line vasopressor superior to dopamine for treatment of septic shock [17].

Steroids may be used for those who are on chronic steroid therapy or adrenal dysfunction, but there is no clear evidence for use low dose steroid to prevent or reduce sepsis and septic shock in critically ill patients, a recent large multicenter RCT demonstrated no reduction in the development of septic shock in septic patients treated with hydrocortisone versus placebo [18].

Patients with sepsis and multiple organ system failure have a high mortality rate, although the outcome of intensive care treatment in critically ill patients may be difficult to prognosis accurately, establishing realistic ICU treatment goals is paramount [19].

The aim of the current study is to investigate the management and outcome of sepsis at our local hospital and how it compares with the international standards for sepsis management.

\section{PATIENTS AND METHODS:}

A prospective observational study carried out on 50 consecutive patients of all age groups regardless of gender with signs of life at presentation from the $1^{\text {st }}$ of February 2017 to the $31^{\text {st }}$ of January 2018. All patients who were admitted to RozhHalat emergency hospital with suspected sepsis were recruited and patient who did not give consent were excluded. Data recorded on a specially designed questionnaire, collected and entered in the computer and then analyzed using appropriate database system which is called Statistical Package for Social Science (SPSS) version 22 and the result were compared between patients with different variables, with a statistical significance level of $<0.05$. The results will be presented as rates, ratio, frequencies, and percentages in tables. The chi square test was performed to compare between the groups.

This study was granted approval by the Ethical and Research Committee of Kurdistan Board of medical specialties. Permission to access patient data was obtained from hospital manager and written Informed consent of permission was also obtained from all participants or their next of kin if patients did not have capacity to give consent.

\section{RESULTS}

Overall mortality was high as $34 / 50(68 \%)$ that shows in figure (1).

As shown in figure (2) the total study group consisted of 50 consequential patients, 21(42\%) male and $29(58 \%)$ female with the male to female ratio of $(2: 3)$. patients died in hospital $13(38.2 \%)$ were male and $21(61.8 \%)$ were female.

Figure (3) shows age distribution which the mean age of the participants was $(47.14 \pm 22.71 \mathrm{SD})$ years old ranging from 10-80 years and the majority of patients $60 \%$ $(30 / 50)$ were above the age of 40 years old.

Figure 1: Outcome

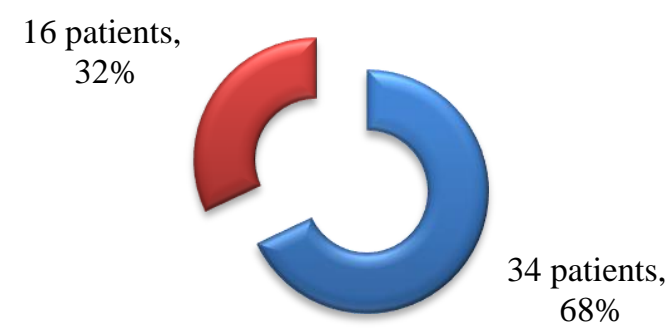


Figure 2: Gender Distribution

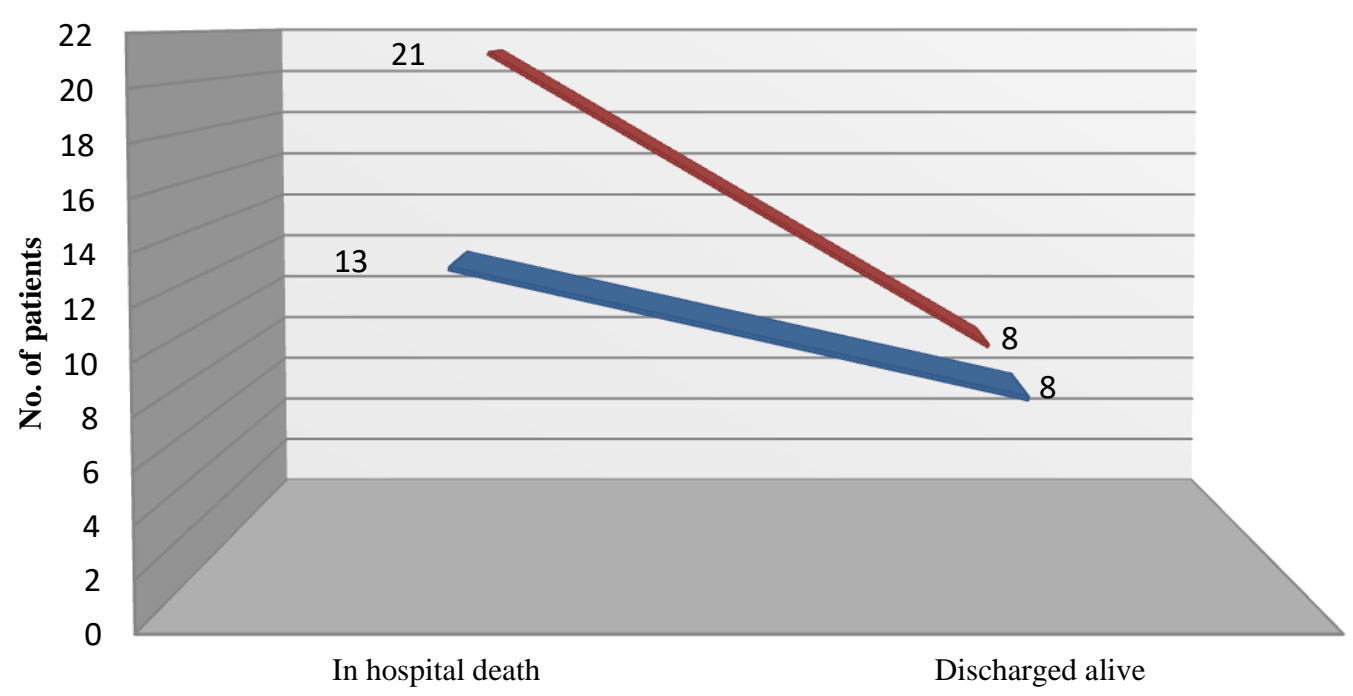

$\square \operatorname{Male}(42 \%)$

$\square$ Female $(58 \%)$

Figure 3: Age distribution

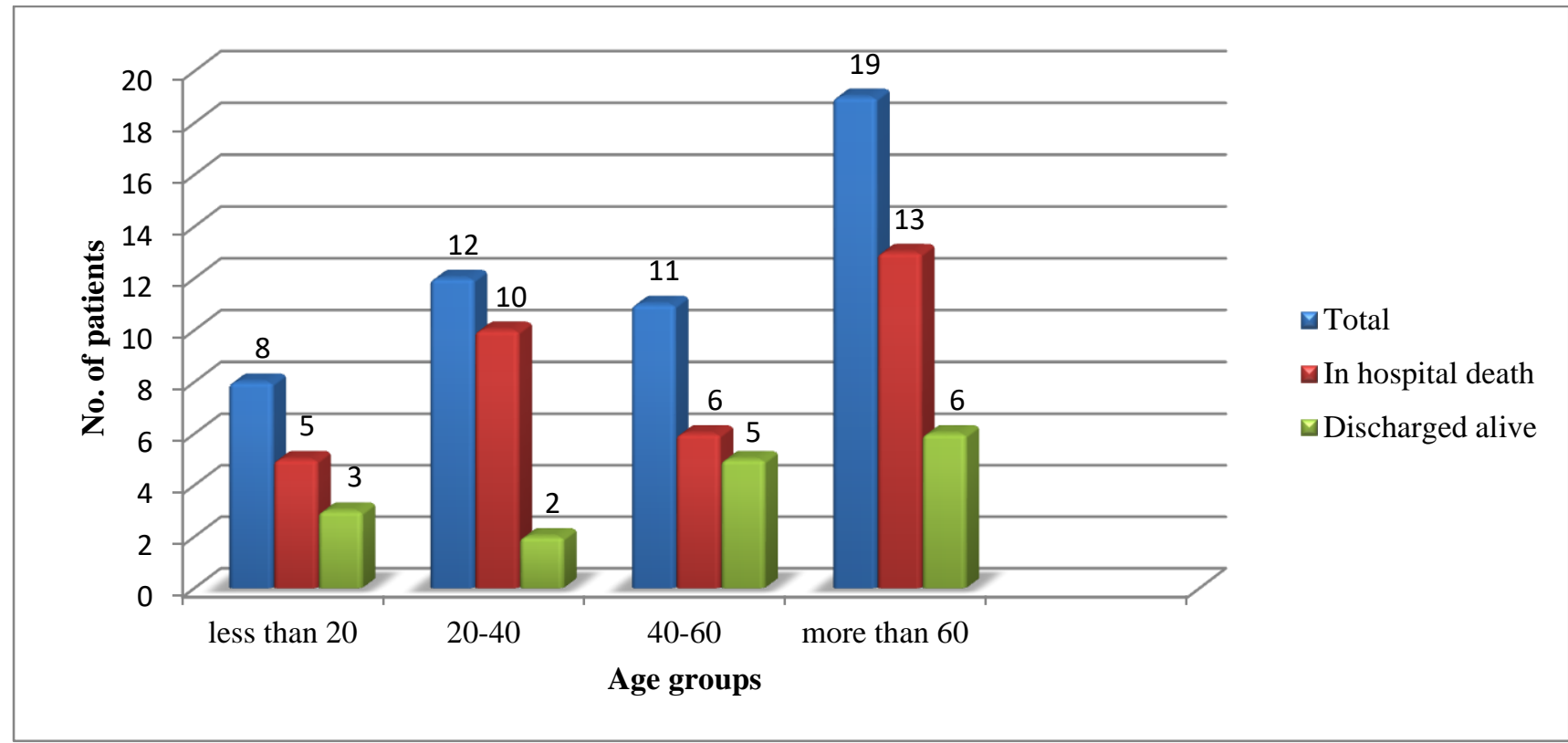

As shown in table (1) the qSOFA was as good as SIRS criteria in predicting mortality. A qSOFA score of more than 2 associated with $86.4 \%$ of mortality compared with $75.5 \%$ mortality with SIRS score of more than 2.A score of less than 1 for both qSOFA and SIRS criteria were associated with low mortality rate of $5.9 \%$ and $0 \%$ respectively. For the qSOFA the highest mortality of $55.9 \%$ was with score of 2 while the highest mortality of $47.1 \%$ associated with SIRS score of 3.

Table 1: Scores

\begin{tabular}{|c|c|c|c|c|}
\hline \multirow{3}{*}{\multicolumn{2}{|c|}{ Scores }} & \multirow{2}{*}{\multicolumn{2}{|c|}{ Outcome }} & \multirow{3}{*}{ Total } \\
\hline & & & & \\
\hline & & In hospital death & Discharged alive & \\
\hline \multirow{7}{*}{$\begin{array}{l}\text { qSOFA } \\
\text { p: (0.001) }\end{array}$} & \multirow[t]{2}{*}{0} & 0 & 5 & 5 \\
\hline & & $0.0 \%$ & $31.3 \%$ & $10.0 \%$ \\
\hline & \multirow[t]{2}{*}{1} & 2 & 6 & 8 \\
\hline & & $5.9 \%$ & $37.5 \%$ & $16.0 \%$ \\
\hline & \multirow[t]{2}{*}{2} & 19 & 4 & 23 \\
\hline & & $55.9 \%$ & $25.0 \%$ & $46.0 \%$ \\
\hline & 3 & 13 & 1 & 14 \\
\hline
\end{tabular}




\begin{tabular}{|c|c|c|c|c|}
\hline & & $38.2 \%$ & $6.3 \%$ & $28.0 \%$ \\
\hline \multirow{2}{*}{\multicolumn{2}{|c|}{ Total }} & 34 & 16 & 50 \\
\hline & & $100.0 \%$ & $100.0 \%$ & $100.0 \%$ \\
\hline \multirow{9}{*}{$\begin{array}{l}\text { SIRS Criteria } \\
\text { P: }(0.006)\end{array}$} & & In hospital death & Discharged alive & \\
\hline & \multirow[t]{2}{*}{1} & 0 & 5 & 5 \\
\hline & & $0.0 \%$ & $31.3 \%$ & $10.0 \%$ \\
\hline & \multirow[t]{2}{*}{2} & 12 & 5 & 17 \\
\hline & & $35.3 \%$ & $31.3 \%$ & $34.0 \%$ \\
\hline & \multirow[t]{2}{*}{3} & 16 & 5 & 21 \\
\hline & & $47.1 \%$ & $31.3 \%$ & $42.0 \%$ \\
\hline & \multirow[t]{2}{*}{4} & 6 & 1 & 7 \\
\hline & & $17.6 \%$ & $6.3 \%$ & $14.0 \%$ \\
\hline \multirow{2}{*}{\multicolumn{2}{|c|}{ Total }} & 34 & 16 & 50 \\
\hline & & $100.0 \%$ & $100.0 \%$ & $100.0 \%$ \\
\hline
\end{tabular}

Lactic acid measured for all patients. Table (2) shows that in 30 patients have lactic acid below $2-\mathrm{mmol} /$ liter, among this group mortality rate was $(63 \%)$. While 20 patients have lactate $2 \mathrm{mmol} / \mathrm{liter}$ and more which 15 patients and mortality was $75 \%$.

Table 2: Lactate level

\begin{tabular}{|l|c|c|c|}
\hline \multirow{2}{*}{$\begin{array}{l}\text { Lactic acid } \\
\text { (P: } 0.06)\end{array}$} & \multicolumn{2}{|c|}{ Outcome } & \multirow{2}{*}{ Total } \\
\cline { 2 - 4 } & In hospital death & Discharged alive & 3 \\
\cline { 2 - 4 } & 0 & 3 & $6.0 \%$ \\
\hline \multirow{2}{*}{$0.9-1.9$} & $0.0 \%$ & $18.8 \%$ & 27 \\
\cline { 2 - 4 } & 19 & 8 & $54.0 \%$ \\
\hline \multirow{2}{*}{$2-4$} & $55.9 \%$ & $50.0 \%$ & $38.0 \%$ \\
\hline \multirow{3}{*}{$>4$} & 14 & 5 & 1 \\
\cline { 2 - 4 } & $41.2 \%$ & 0 & $2.0 \%$ \\
\hline Total & 1 & $0.0 \%$ & 50 \\
& $2.9 \%$ & 16 & $100.0 \%$ \\
\hline
\end{tabular}

Blood culture was done for all patients by the main investigator and in 30/50 (60\%) it was taken before patients received Antibiotics. The blood culture result did not grow any organism in $13(26 \%)$ of patients, while it was positive in the remaining $37(74 \%)$ patients of which $26 / 50(52 \%)$ grew gram positive and $11 / 50$ (22\%) gram-negative organism.
The commonest gram-positive organism was streptococcus (24\%) followed by staphylococcus (22\%) while the E. Coli (12\%) was the most comment gramnegative infective agent. The overall mortality rate for gram-positive organism was $84.6 \%$ compared with $72 \%$ for gram-negative causative agents while mortality was lower $(31 \%)$ if the blood culture was negative.

Table 3: Blood culture and microorganisms

\begin{tabular}{|c|c|c|c|}
\hline \multirow[b]{2}{*}{$\begin{array}{l}\text { Microorganisms } \\
\text { (P:0.04) }\end{array}$} & \multicolumn{2}{|c|}{ Outcome } & \multirow[t]{2}{*}{ Total } \\
\hline & In hospital death & Discharged alive & \\
\hline \multirow[t]{2}{*}{ Negative blood culture } & 4 & 9 & 13 \\
\hline & $11.8 \%$ & $56.3 \%$ & $26.0 \%$ \\
\hline \multirow[t]{2}{*}{ G+ staphylococcus } & 9 & 2 & 11 \\
\hline & $26.5 \%$ & $12.5 \%$ & $22.0 \%$ \\
\hline \multirow[t]{2}{*}{ G+ streptococcus } & 11 & 1 & 12 \\
\hline & $32.4 \%$ & $6.3 \%$ & $24.0 \%$ \\
\hline \multirow[t]{2}{*}{ G+ enterococcus } & 2 & 1 & 3 \\
\hline & $5.9 \%$ & $6.3 \%$ & $6.0 \%$ \\
\hline \multirow[t]{2}{*}{ G- E.coli } & 4 & 2 & 6 \\
\hline & $11.8 \%$ & $12.5 \%$ & $12.0 \%$ \\
\hline \multirow[t]{2}{*}{ G- Klebsiella } & 3 & 1 & 4 \\
\hline & $8.8 \%$ & $6.3 \%$ & $8.0 \%$ \\
\hline
\end{tabular}




\begin{tabular}{|l|c|c|c|}
\hline \multirow{2}{*}{ G- Acinetobacter } & 1 & 0 & 1 \\
\cline { 2 - 4 } & $2.9 \%$ & $0.0 \%$ & $2.0 \%$ \\
\hline \multirow{2}{*}{ Total } & 34 & 16 & 50 \\
\cline { 2 - 4 } & $100.0 \%$ & $100.0 \%$ & $100.0 \%$ \\
\hline
\end{tabular}

Regarding the site of infection (figure 4) the commonest site of infection was from multiple sources (34\%) with a high mortality of $47.1 \%$, the second most common site was the respiratory source $(26 \%)$ with the overall mortality of $(29.4 \%)$. The skin and soft tissue sepsis was associated with the lowest mortality rate of $2.9 \%$

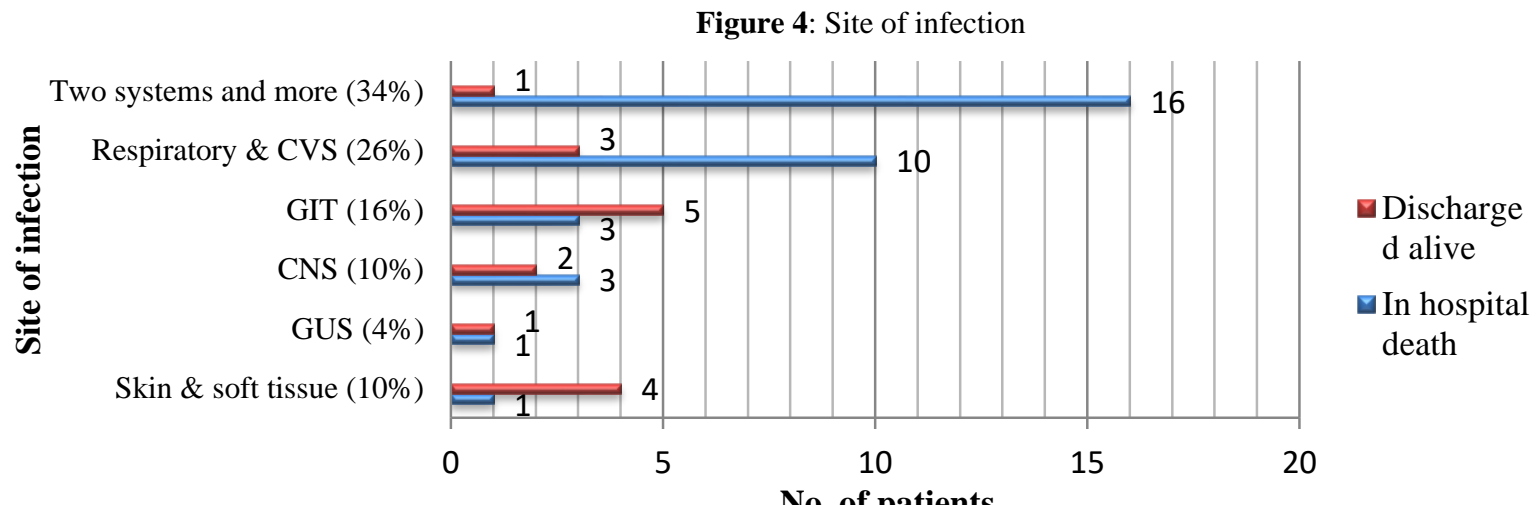

All patients received empirical antibiotic therapy however $20 / 50(40 \%)$ received antibiotics before obtaining the blood culture.

The patients who received the right empirical antibiotics $27 / 50(54 \%)$ they had a lower mortality rate
$(55.5 \%)$ compared with $82 \%$ mortality if they did not receive the correct empirical antibiotics 23/50 (46\%). Likewise, if the antibiotics were changed correctly after blood culture results the mortality were lower $(58.6 \%)$ compared with $80 \%$ chance of death if the antibiotics were not changed.

Table 4: Empirical antimicrobials

\begin{tabular}{|c|c|c|c|}
\hline \multirow[b]{2}{*}{ Right Empirical antibiotic (P:0.8) } & \multicolumn{2}{|c|}{ Outcome } & \multirow[t]{2}{*}{ Total } \\
\hline & In hospital death & Discharged alive & \\
\hline \multirow[t]{2}{*}{ Yes } & 15 & 12 & 27 \\
\hline & $44.1 \%$ & $75.0 \%$ & $54.0 \%$ \\
\hline \multirow[t]{2}{*}{ No } & 19 & 4 & 23 \\
\hline & $55.9 \%$ & $25.0 \%$ & $46.0 \%$ \\
\hline \multirow[t]{2}{*}{ Total } & 34 & 16 & 50 \\
\hline & $100.0 \%$ & $100.0 \%$ & $100.0 \%$ \\
\hline $\begin{array}{l}\text { Antibiotic change after } \\
\text { result of blood Culture }\end{array}$ & In hospital death & Discharged alive & \\
\hline \multirow[t]{2}{*}{ Yes } & 17 & 12 & 29 \\
\hline & $50.0 \%$ & $75.0 \%$ & $58.0 \%$ \\
\hline \multirow[t]{2}{*}{ No } & 17 & 4 & 21 \\
\hline & $50.0 \%$ & $25.0 \%$ & $42.0 \%$ \\
\hline \multirow[t]{2}{*}{ Total } & 34 & 16 & 50 \\
\hline & $100.0 \%$ & $100.0 \%$ & $100.0 \%$ \\
\hline
\end{tabular}

Table (5) reveal that fluid therapy (crystalloid) used at two pattern, the bolus fluid therapy in the first one hour of admission and maintenance fluid in the first 24 hour of admission.

Among 47 of patients who received more than one litter maintenance crystalloid fluids in the first hour, 16 patients $(34 \%)$ discharged alive. While none of the 3 patients who received less than one litter of maintenance fluids were discharged alive

Table 5: Fluid therapy

\begin{tabular}{|c|c|c|c|}
\hline \multirow{2}{*}{$\begin{array}{l}\text { Bullous dose of IV Fluid } \\
(\mathrm{P}: 0.24)\end{array}$} & \multicolumn{2}{|c|}{ Outcome } & \multirow[t]{2}{*}{ Total } \\
\hline & In hospital death & Discharged alive & \\
\hline \multirow[t]{2}{*}{$<250 \mathrm{ml}$} & 0 & 1 & 1 \\
\hline & $0.0 \%$ & $6.3 \%$ & $2.0 \%$ \\
\hline
\end{tabular}




\begin{tabular}{|c|c|c|c|}
\hline \multirow[t]{2}{*}{$250-500 \mathrm{ml}$} & 26 & 13 & 39 \\
\hline & $76.5 \%$ & $81.3 \%$ & $78.0 \%$ \\
\hline \multirow[t]{2}{*}{$500-1000 \mathrm{ml}$} & 8 & 2 & 10 \\
\hline & $23.5 \%$ & $12.5 \%$ & $20.0 \%$ \\
\hline \multirow[t]{2}{*}{ Total } & 34 & 16 & 50 \\
\hline & $100.0 \%$ & $100.0 \%$ & $100.0 \%$ \\
\hline $\begin{array}{l}\text { Maintenance dose of } \\
\text { IV Fluid } \quad(\mathrm{P}: 0.3)\end{array}$ & In hospital death & Discharged alive & Total \\
\hline \multirow[t]{2}{*}{$0.5-1$ lit. } & 3 & 0 & 3 \\
\hline & $8.8 \%$ & $0.0 \%$ & $6.0 \%$ \\
\hline \multirow[t]{2}{*}{$1-2$ lit. } & 15 & 10 & 25 \\
\hline & $44.1 \%$ & $62.5 \%$ & $50.0 \%$ \\
\hline \multirow[t]{2}{*}{$>2$ lit. } & 16 & 6 & 22 \\
\hline & $47.1 \%$ & $37.5 \%$ & $44.0 \%$ \\
\hline \multirow[t]{2}{*}{ Total } & 34 & 16 & 50 \\
\hline & $100.0 \%$ & $100.0 \%$ & $100.0 \%$ \\
\hline
\end{tabular}

Majority of patients 36/50 (72\%) received vasopressor therapy and 14/50 (28\%) did not receive inotropics. The overall mortality among vasopressor treated patients were $72 \%$ compared with $78 \%$ chance of death if they were not treated with any vasopressor therapy. Noradrenaline therapy associated with the highest survival rate $(72 \%)$ while combined inotropic use associated with the lowest survival rate.

Table 6: Vasopressors

\begin{tabular}{|c|c|c|c|}
\hline \multirow{2}{*}{$\begin{array}{l}\text { Vasopressor } \\
\text { (P: } 0.005)\end{array}$} & \multicolumn{2}{|c|}{ Outcome } & \multirow[t]{2}{*}{ Total } \\
\hline & In hospital death & Discharged alive & \\
\hline \multirow[t]{2}{*}{ Not received } & 11 & 3 & 14 \\
\hline & $32.4 \%$ & $18.8 \%$ & $28.0 \%$ \\
\hline \multirow{2}{*}{ Noradrenaline } & 3 & 8 & 11 \\
\hline & $8.8 \%$ & $50.0 \%$ & $22.0 \%$ \\
\hline \multirow[t]{2}{*}{ Dopamine } & 13 & 4 & 17 \\
\hline & $38.2 \%$ & $25.0 \%$ & $34.0 \%$ \\
\hline \multirow[t]{2}{*}{ Dobutamine } & 0 & 1 & 1 \\
\hline & $0.0 \%$ & $6.3 \%$ & $2.0 \%$ \\
\hline \multirow{2}{*}{ Combination } & 7 & 0 & 7 \\
\hline & $20.6 \%$ & $0.0 \%$ & $14.0 \%$ \\
\hline \multirow[t]{2}{*}{ Total } & 34 & 16 & 50 \\
\hline & $100.0 \%$ & $100.0 \%$ & $100.0 \%$ \\
\hline
\end{tabular}

Figures $(5,6)$ shows the rate of complications in this study group. Majority of cases (82\%) developed organ failure. The highest overall mortality rate $(73.5 \%)$ was associated with more than 2 organ failures. If there were no complications the survival rate was $88.8 \%(8 / 9$ cases).

Figure 5: Type of complications

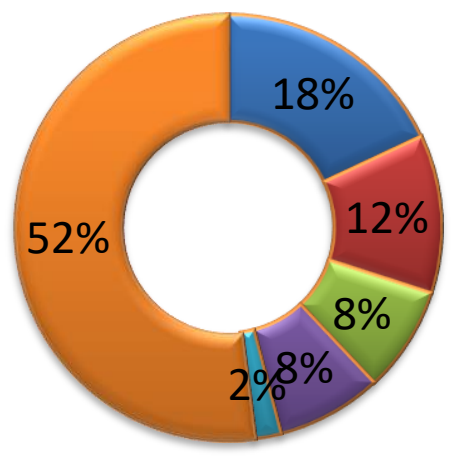

\footnotetext{
No complication(9 patients)

- Renal failure (6 patients)

$\nabla$ Respiratory failure (4 patients)

- Liver failure (4 patients)

ఐHeart failure (1 patient)

$\nabla$ Two systems \& more failure (26 patients)
}

Figure 6: Outcome of complications 


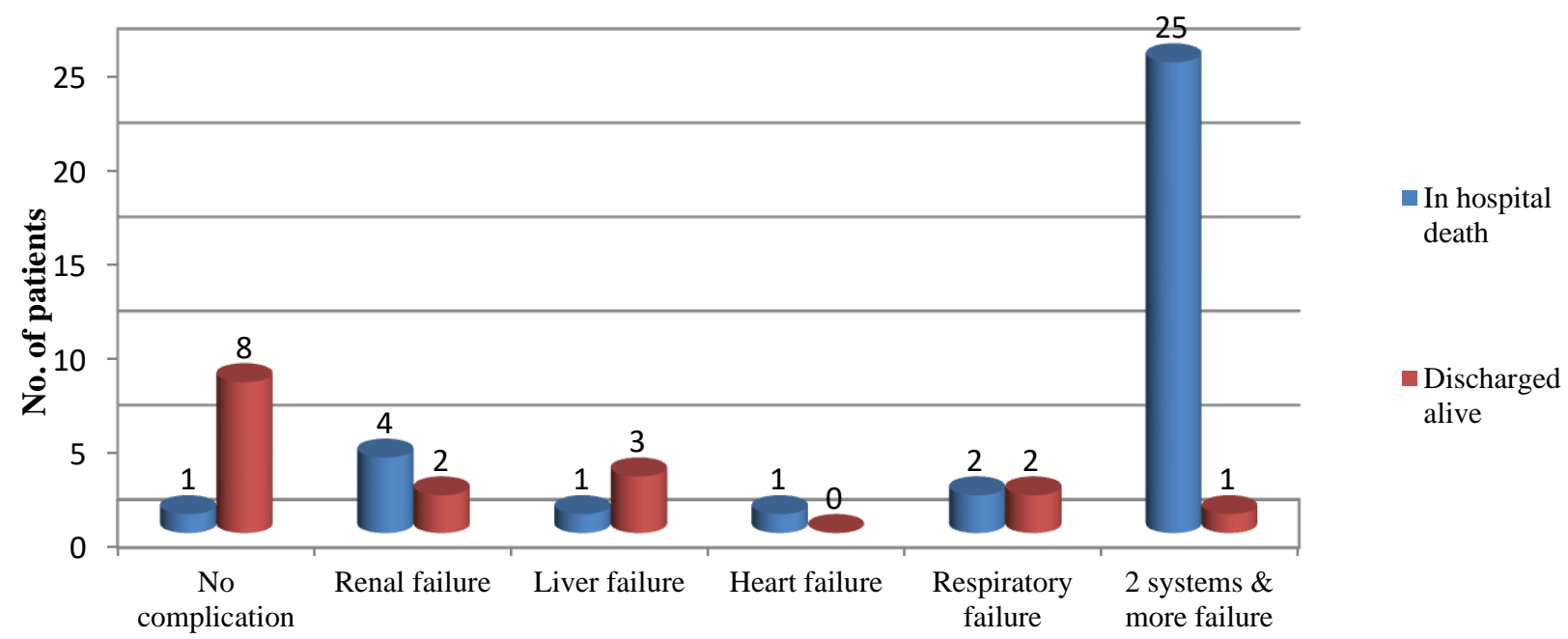

Compications

\section{DISCUSSION}

Sepsis is a serious consequence of infection; it has a high mortality rate worldwide. Septic shock is the most severe and life-threatening complication of sepsis that has irreversible damage that could end up with multi organ dysfunction and death, therefore early recognition and management of septic patients is crucial to reduce mortality rate [5].

The new sepsis definition is designed to identify patients with sepsis with easier criteria's such as qSOFA and categories sepsis into three new main groups of: infection, sepsis and septic shock. Sever sepsis deemed not to be relevant according to the new consensus of sepsis guideline [5].

Adherence to the sepsis 6 bundle of resuscitation is very important to treat sepsis with effective treatment to reduce mortality and morbidity from all categories of sepsis [8].

Multiple international studies revealed that sepsis is more common in the older age group, a study reported in 2006 in the United States shows that $64.9 \%$ of patients with sepsis aged more than 65 years of age [20]. Another study reported in India in 2012 shows that severe sepsis and septic shock in elderly patients are not only more common but also associated with higher mortality rate [21], In this study $38 \%$ of patients were aged more than 60 years old resulted in $68.4 \%$ of mortality.

In this study female patients were affected more than male to have sepsis and also mortality rate was higher in females compared to male $(68.2 \%$ and 31.85 respectively) [22] [23].

Three separate studies done in 2017, (USA, Brazil and South Korea) found that qSOFA provided better discrimination than SIRS criteria for predicting mortality. In this study the qSOFA score higher than 2 associated with $86.4 \%$ mortality, while similar SIRS criteria score resulted in $75.5 \%$ mortality [24] [25] [26]. Serum lactic acid measurement has a predicting rule for mortality in sepsis and recommended in first hour for early recognition of sepsis. In our study mortality rate was high $(75 \%)$ for those patients whom serum lactate level more than $2 \mathrm{mmol} / \mathrm{liter}$, this result is comparable to other studies done in Sao Paulo brazil in 2016 [27], Vancouver Canada in 2012 [28] and Seoul south Korea in 2011 [29].

In our study $26 \%$ have negative blood culture which was lower compared to other studies done in Singapore $(41.5 \%)$ in 2013 [30] and in Spain (35\%) in 2008 [31].

Higher mortality rate is seen among gram positive causative microorganism which was $67.4 \%$ in this study, a similar result is seen in a study reported in Denmark in 2011 [32].

The finding of this study shows that respiratory infection where the second most common source $(26 \%)$ for septic patients and mortality rate in patient with respiratory source sepsis was $29.4 \%$.In $34 \%$ of patients two or more systems were infected and in those patients mortality rate was very high(47.1\%), these results comparable to a similar studies done in Denmark in 2016 [33] and another carried out in Pennsylvania U.S.A. in 2014 [34]. Empirical antimicrobial therapy was proved to be inappropriate among $46 \%$ of patient recorded in our study with a high mortality rate of $82.6 \%$. While those with appropriate antimicrobial therapy (54\%), mortality rate was less $(55.5 \%)$, this finding is identical to a study done in Thailand in 2012 [35].

Following blood culture results the antimicrobial therapy were changed in some patients which resulted with lower mortality rate of $58.6 \%$, compared with $80 \%$ mortality if the antibiotics were not changed, a study done in Indonesia in 2013 revealed that appropriate empirical antibiotic has better result in outcome for septic patients [36].

In our study the mortality rate was not significantly different if they received vasopressor therapy or not (72\% versus $78 \%$ ). In patients who received noradrenaline as a vasopressor they had higher survival rate $(72.7 \%)$ compared to those whom received dopamine $(23.5 \%)$, same result reported in studies done in Belgium in 2012 [37] and in Washington U.S.A in 2015 [38].

Failure of two or more vital organ systems is termed multi-organ dysfunction syndrome and associated with 
high mortality. In this study $34 \%$ of patients had two or more organ dysfunction and mortality rate among them were $94 \%$. Respiratory failure was detected in $26 \%$ of patients and with mortality rate $(76.9 \%)$, which was highest rate in compare to other study [39].

High mortality rates for sepsis and septic shock in our study was $(68 \%)$, This figure also has been in other studies in other middle and low-income countries, the overall mortality rate of severe sepsis was $(55.7 \%)$ in Brazil in 2015 [40], (64.6\%) in India in 2007 [41] and (49.7\%) in Thailand in 2009 [42].

The poor survival rate in developing countries related to many factors such as lack of resources, lack of standardized sepsis local protocol, unavailability of blood cultures, and lack of awareness. In the hospital that this study was carried out all these factors were present that would explain higher mortality rate compared with the developed countries.

We hope that these findings will lead to development of local and national protocol in the Kurdistan region of

\section{REFERENCES}

[1] L.-Z. W. L. J. C. G. C. J. P. M. Angus DC, "Epidemiology of severe sepsis in the United States: analysis of incidence, outcome, and associated costs of care," Society of Critical Care Medicine and Lippincott Williams, vol. 29, no. 7, pp. 1303-1310, 2001.

[2] E. G. G. J. S. J. W. A. A. D. I. T. Liu V, "Hospital deaths in patients with sepsis," Hospital deaths in patients with sepsis from 2 independent cohorts. JAMA, pp. 90-92, 2014.

[3] R. S. M. L. L. O. S. M. R. K. T. I. R. \&. V. J.-L. Hotchkiss, "Sepsis and septic shock.," Nature reviews Disease primers., pp. 16-45, 2016.

[4] "National Institute for Health and Care Excellence. Sepsis: recognition, diagnosis and early management.," 175 2017. [Online] Available: https://www.nice.org.uk. [Accessed 137 2016].

[5] M. D. C. S. S. C. W. S.-H. M. A. D. B. M. ... A. D. C. (. Singer, "The Third International Consensus Definitions for Sepsis and Septic Shock," Sepsis-3. JAMA., vol. 315(8), pp. 801-810, 2016.

[6] C. W. L. V. X. I. T. J. B. F. M. R. T. D. S. A. Seymour, "Assessment of Clinical Criteria for Sepsis," Third International Consensus Definitions for Sepsis and Septic Shock, vol. 315(8), pp. 762-774, 2016

[7] M. M. Z. F. J. W. C. H. M. D. \&. E. D. P. Churpek, "Incidence and Prognostic Value of the Systemic Inflammatory Response Syndrome and Organ Dysfunctions in Ward Patients," American Journal of Respiratory and Critical Care Medicine, vol. 192(8), pp. 958-964, 2015.

[8] N. T. M. G. Daniels R, "The sepsis six and the severe sepsis resuscitation bundle, a prospective observational cohort study," Emergency medicine journal: EMJ., vol. 28(6), pp. 507-512, 2010.

[9] P. K. O. M. A. R. M. M. D. V. V. E. S. D. M. S. E. M. B. \&. d. S O. F. P. Yokota, "Impact of Appropriate Antimicrobial Therapy for Patients with Severe Sepsis and Septic Shock," A Quality Improvement Study, pp. 9-11, 2014.

[10] M.-L. I. P. G. Ferrer R, "Empiric antibiotic treatment reduces mortality in severe sepsis and septic shock," guideline-based performance improvement program., vol. 42(8), pp. 1749-1755, 2014.

[11] F. R. P. R. R. G. E. P. B. B. M. J. M. G. K. S. L. D. R. D. K. A. Amaral AC, "Patient and Organizational Factors Associated With Delays in Antimicrobial Therapy for Septic Shock.," Antimicrobial Therapy of Septic Shock, vol. 44(12), pp. 2145-2153, 2016.

[12] E. P. A. Y. Kumar A, "Initiation of inappropriate antimicrobial therapy results in a fivefold reduction of survival in human septic shock.," Antimicrobial Therapy of Septic Shock, vol. 136(5), pp. 1237-1248, 2009.
Iraq to manage sepsis with a better standard of care that will lead to reduction of mortality from such a common and deadly condition [43].

\section{CONCLUSION}

This study shows that sepsis is associated with high overall mortality rate of $(68 \%)$ in the RozhHalat emergency hospital and higher rate of death among female and older age group. Negative blood culture, appropriate use of antibiotics and fluid therapy associated with better chance of survival. Vasopressor therapy did not result in better survival outcome except for Noradrenaline.

The qSOFA score is as good as SIRS criteria in predicting mortality. High lactate, multi-organ failure and multisource of infection associated with the worst outcome.

[13] M. W. \&. R. T. W. Semler, "Sepsis Resuscitation: Fluid Choice and Dose," Clinics in Chest Medicine, vol. 37(2), pp. 241-250, 2016.

[14] R. A. P. G. Levy MM, "association between performance metrics and outcomes in a 7.5-year study," Surviving Sepsis Campaign, vol. 43(1), pp. 3-12, 2015.

[15] P. G. S. C. e. a. Casserly B, "Lactate measurements in sepsisinduced tissue hypoperfusion," Surviving Sepsis Campaign database, vol. 43(3), pp. 567-573, 2015.

[16] T. H. H. Z. e. a. Yu B, "Comparison of the effect of fluid resuscitation as guided either by lactate clearance rate or by central venous oxygen saturation in patients with sepsis.," vol. 25(10), pp. 578-583, 2013.

[17] T. L. A. L. S. L. L. P. M. \&. G. A. Avni, "Vasopressors for the Treatment of Septic Shock," 38 2015. [Online]. Available: http://doi.org/10.1371/journal.pone.0129305.

[18] T. E. M. G. e. a. Keh D, "Effect of hydrocortisone on development of shock among patients with severe sepsis," HYPRESS randomized clinical trial. JAMA, vol. 316(17), pp. 1775-1782, 2016.

[19] E. R. W. M. White DB, "The language of prognostication in intensive care units," An International Journal of the Society for Medical Decision Making, vol. 30(1), pp. 76-83, 2010.

[20] M. D. M. M. M. Martin G. S., "The effect of age on the development and outcome of adult sepsis.," vol. 34(1), pp. 15-21, 2006.

[21] J. D. S. O. Nasa P, "Severe sepsis and septic shock in the elderly," World Journal of Critical Care Medicine, vol. 1(1), pp. 23-30, 2012.

[22] E. C. M. L. e. a. Sakr Y, "The influence of gender on the epidemiology of and outcome from severe sepsis," 1832013. [Online]. Available: https://ccforum.biomedcentral.com/articles/10.1186/cc12570.

[23] S. N. L. P. e. a. Fowler RA, "Sex-and age-based differences in the delivery and outcomes of critical care," Canadian Medical Association Journal., vol. 177(12), pp. 1513-1519, 2007.

[24] J. D. M. K. e. a. Finkelsztein EJ, "Comparison of qSOFA and SIRS for predicting adverse outcomes of patients with suspicion of sepsis outside the intensive care unit.," vol. 21, p. 73, 2017.

[25] G. J. S. J. A. Serafim R, "comparison of the quick-SOFA (qSOFA) and SIRS criteria for the diagnosis of sepsis and prediction of mortality : A Systematic Review and Meta-Analysis," American College of Chest Physicians, vol. 153, pp. 646-655, 2017.

[26] K. W. K. M. e. a. Park HK, "Quick sequential organ failure assessment compared to systemic inflammatory response syndrome for predicting sepsis in emergency department," Journal of Critical Care, vol. 42, pp. 12-17, 1962017.

[27] R. L. C. T. e. a. Filho RR, "Blood Lactate Levels Cutoff and Mortality Prediction in Sepsis-Time for a Reappraisal? a Retrospective Cohort Study," vol. 46(5), pp. 480-485, 5112016. 
[28] N. T. B. J. e. a. Wacharasint P, "Normal-range blood lactate concentration in septic shock is prognostic and predictive," vol. 38, pp. 1:4-10, 172012.

[29] U. S. K. W. e. a. Kang YR, "Initial lactate level and mortality in septic shock patients with hepatic dysfunction.," vol. 39, no. 5, pp. 862-867, 2011

[30] N. W. S. K. e. a. Phua J, "Characteristics and outcomes of culturenegative versus culture-positive severe sepsis," vol. 17, no. 5, 129 2013.

[31] M.-B. A. S. V. e. a. Blanco J, "Incidence, organ dysfunction and mortality in severe sepsis: a Spanish multicentre study," vol. 12, no. 6, 17122008 .

[32] N. M. P. L. e. a. Sogaard M, "Blood culture status and mortality among patients with suspected community acquired bacteremia: a population-based cohort study," vol. 11, p. 139, 2052011.

[33] A. M. J. M. e. a. Vibeke K, "Site of infection and mortality in patients with severe sepsis or septic shock:A cohort study of patients admitted to a Danish general intensive care unit, .," Infectious Diseases Journal, vol. 48, no. 10, pp. 726-731, 872016.

[34] Y. S. A. D. Mayr FB, "Epidemiology of severe sepsis," Journal Virulence, vol. 5, no. 1, pp. 4-11, 1112201.

[35] L. A. Lueangarun S, "Impact of Inappropriate Empiric Antimicrobial Therapy on Mortality of Septic Patients with Bacteremia: A Retrospective Study," Interdisciplinary Perspectives on Infectious Diseases, pp. 1-13, 2852012.

[36] S. D. L. K. e. a. Pradipta IS, "Antibiotic Resistance in Sepsis Patients: Evaluation and Recommendation of Antibiotic Use.," North American Journal of Medical Sciences, vol. 5, no. 6, pp. 344 352, 2013.

[37] A. C. N. H. V. J. De Backer D, "Dopamine versus norepinephrine in the treatment of septic shock," a meta-analysis. Crit Care Med., vol. 40, pp. 725-730, 132012.

[38] E. S. A. C. Pollard S, "Vasopressor and Inotropic Management Of Patients With Septic Shock.," Pharmacy and Therapeutics., vol. 40, no. 7, pp. 438-450, 2015.

[39] H. S. Kim WY, "Sepsis and Acute Respiratory Distress Syndrome: Recent Update.," Tuberculosis and Respiratory Diseases., vol. 79, no. 2, pp. 53-57, 2016.

[40] B. C. B. F. e. a. Machado FR, "Epidemiology of sepsis in Brazilian ICUs,a nationwide stratified sample.," Intensive Care Medicine Experimental, vol. 3, no. 1, 2015

[41] C. S. B. M. Todi S, "Epidemiology of severe sepsis in India," Critical Care, vol. 11, no. 2, p. 65, 2007.

[42] B. R. Khwannimit B, "The epidemiology of, and risk factors for mortality from severe sepsis and septic shock in a tertiary care university hospital setting," Cambridge University Press, vol. 137, no. 9, pp. 1333-1341, 422009.

[43] S. M. B. R. e. a. Dagher GA, "Descriptive analysis of sepsis in a developing country," International Journal of Emergency Medicine, vol. 8, no. 19,662015 . 